\section{0-205 PARENTAL CARDIOVASCULAR MORBIDITY IN FAMILIES WITH A PRETERM CHILD, A NATIONAL REGISTER STUDY}

${ }^{1} \mathrm{P}$ Hovi, ${ }^{1} \mathrm{~S}$ Turkka, ${ }^{1} \mathrm{SPK}$ Näsänen-Gilmore, ${ }^{2} \mathrm{M}$ Vääräsmäki, ${ }^{3} \mathrm{M}$ Gissler, ${ }^{4} \mathrm{~A}$ Pouta, ${ }^{5} \mathrm{E}$ Kajantie. ${ }^{1}$ Department of Chronic Disease Prevention, National Institute for Health and Welfare (THL), Helsinki, Finland; ' ${ }^{2}$ Department of Obstetrics and Gynecology, Oulu University Hospital, Oulu, Finland; ${ }^{3}$ Information Department, National Institute for Health and Welfare, Helsinki, Finland; ${ }^{4}$ Department of Children Young People and Families, National Institute for Health and Welfare, Oulu, Finland; ${ }^{5}$ Department of Chronic Disease Prevention, National Institute for Health and Welfare, Helsinki, Finland

\subsection{6/archdischild-2014-307384.275}

Background Prematurity is associated with a higher incidence of hypertension and glucose metabolism during childhood and young adulthood. This may be due to circumstances during fetal and early postnatal life or to confounders such as unhealthy lifestyle in childhood home. The confounder explanation would gain support if preterm birth would predict father's cardiovascular disease (CVD).

Objective We hypothesised that preterm birth would predict cardiovascular disease in both parents.

Design/methods Pregnancy data came from Finnish Medical Birth Register. During Jan 1, 1987 and Sep 30, 1990, we included the first singleton birth for each of the 196,427 mothers. National Population Register Centre tracked the fathers, 3,048 remained missing (and 27 mothers).

We obtained Hospital Discharge Register data from Aug, 1969, and non-primary care out-patient visit data from Sep 1986, both until 31 Dec 2012. The first occasion of CVD was when the ICD-9 and ICD-10 indicated coronary heart disease (CHD) or stroke.

We utilised Cox regression for proportional hazards to analyse the effect of GA group on CVD in mothers and fathers separately.

Results In mothers, preterm birth predicted CVD with Hazard Ratios (HRs) increasing up to 2.12 with shorter gestation (Table 1). Among thefathers, those whose baby had been born preterm were clearly not at ahigher risk for a CVD diagnosis (Table 2).

Abstract 0-205 Table 1 Mohter's CVD diagnosis by index child's gestational age group

\begin{tabular}{|c|c|c|c|c|c|}
\hline \multirow{2}{*}{$\begin{array}{l}\text { Group* } \\
<28\end{array}$} & \multirow{2}{*}{$\frac{\mathrm{HR}}{2.00}$} & \multicolumn{2}{|c|}{$95 \% \mathrm{Cl}$ for HR } & \multirow{2}{*}{$\begin{array}{r}n \\
417\end{array}$} & \multirow{2}{*}{$\begin{array}{r}\text { CVD } \\
26\end{array}$} \\
\hline & & 1.36 & 2.94 & & \\
\hline 28 to 31 & 2.12 & 1.61 & 2.79 & 809 & 52 \\
\hline 32 to 33 & 1.61 & 1.22 & 2.13 & 954 & 50 \\
\hline 34 to 36 & 1.55 & 1.38 & 1.74 & 6540 & 303 \\
\hline 37 to 38 & 1.26 & 1.18 & 1.34 & 32996 & 1272 \\
\hline 39 to 41 & 1.00 & & & 143499 & 4127 \\
\hline$>41$ & 0.95 & 0.83 & 1.09 & 8587 & 213 \\
\hline Missing & 0.98 & 0.78 & 1.23 & 2598 & 74 \\
\hline Total & & & & 196400 & 6117 \\
\hline
\end{tabular}

*full weeks of gestational age

Conclusions Since preterm birth predicted CVD only in mothers and not fathers, the higher CVD risk factors in those born preterm are unlikely to be mediated by unhealthy habits learnt from the parents.
Abstract 0-205 Table 2 Father's CVD diagnosis by index child's gestational age group

Father's CVD diagnosis by index child's gestational age group

\begin{tabular}{lccrrr}
\hline Group* & HR & \multicolumn{2}{c}{$95 \%$ Cl for HR } & n & CVD \\
\hline$<28$ & 0.71 & 0.49 & 1.03 & 347 & 28 \\
28 to 31 & 0.94 & 0.75 & 1.19 & 759 & 72 \\
32 to 33 & 0.67 & 0.53 & 0.84 & 917 & 73 \\
34 to 36 & 0.97 & 0.89 & 1.05 & 6353 & 599 \\
37 to 38 & 0.91 & 0.88 & 0.95 & 32490 & 2936 \\
39 to 41 & 1.00 & & & 141690 & 12094 \\
$>41$ & 1.18 & 1.09 & 1.27 & 8440 & 683 \\
Missing & 0.94 & 0.82 & 1.07 & 2383 & 215 \\
Total & & & & 193379 & 16700 \\
\hline
\end{tabular}

*full weeks of gestational age

\section{0-206 POORER COGNITIVE AND GROSS MOTOR OUTCOME AT AGE 2.5 YEARS AFTER INTRAUTERINE EXPOSURE TO SSRI. PROCEEDINGS FROM THE DUTCH SMOK TRIAL}

${ }^{1} \mathrm{CN}$ van der Veere, ${ }^{2} \mathrm{NKS}$ de Vries, ${ }^{3} \mathrm{AF}$ Bos. ${ }^{1}$ General Pediatrics, Beatrix Children's Hospital/University Medical Center Groningen, Groningen, Netherlands; ${ }^{2}$ General Pediatrics, Medical Center Leeuwarden, Leeuwarden, Netherlands; ${ }^{3}$ Neonatology, Beatrix Children's Hospital/University Medical Center Groningen, Groningen, Netherlands

\subsection{6/archdischild-2014-307384.276}

Background Prescription rates of antidepressant medication during pregnancy range from $2.0 \%$ in the Netherlands to $13.4 \%$ in the USA. Concern has risen about the long-term effects of prenatal exposure to selective serotonin reuptake inhibitors (SSRIs) on the developing child.

Aim Determine the effect of prenatal exposure to SSRIs on neurodevelopment at 2.5 years.

Methods During pregnancy, 107 mother-infant pairs were included, of whom 63 were SSRI-exposed (SSRI-group) and 44 non-exposed (non SSRI). In both groups maternal depression and anxiety were measured using questionnaires (Beck Depression Inventory (BDI) and Spielberger's State Trait Anxiety Inventory (STAI), respectively).

At age 2.5 years 102 infants were tested using the Bailey Scales of Infant Development 3th edition (cognitive, fine motor and gross motor scales). Scaled scores (SS) were used for statistical analysis.

\section{Abstract 0-206 Table 1}

\begin{tabular}{lllll}
\hline & SSRI group & Non SSRI group & Adjusted STAI & Adjusted BDI \\
\hline & SS mean \pm SD & SS mean \pm SD & $\mathrm{p}$ & $\mathrm{p}$ \\
Cognition & $9.0 \pm 1.4$ & $9.9 \pm 1.7$ & 0.001 & 0.004 \\
Fine motor & $10.2 \pm 2.4$ & $10.6 \pm 2.2$ & $\mathrm{NS}$ & $\mathrm{NS}$ \\
Gross motor & $7.9 \pm 2.2$ & $9.1 \pm 2.5$ & 0.036 & 0.003 \\
\hline
\end{tabular}

Results

Conclusions SSRI-exposed children perform significantly worse on the cognitive and gross motor scale of the BSID-III, independent of maternal depression, anxiety, education, smoking and alcohol use during pregnancy. 


\section{0-207 THE STATUS OF EARLY CHILDHOOD DEVELOPMENT OF "LEFT-BEHIND CHILDREN": A CROSS-SECTIONAL SURVEY IN RURAL CHINA}

${ }^{1} \mathrm{H}$ Guan, ${ }^{1} X W$ Tao, ${ }^{1} \mathrm{ZY}$ Fan, ${ }^{2} Y \mathrm{R}$ Zhao. ${ }^{1}$ Early Childhood Development, Capital Institute of Pediatrics, Beijing, China; '2Department of Developing Pediatric, Shengjing Hospital China Medical University, Shenyang, China

\subsection{6/archdischild-2014-307384.277}

Objective Approximately 58 million of children living in rural areas in China have been left behind after their parents migrated to cities to find work. The impacts of parental migration on the early development of left-behind children (LBC) are uncertain. We conducted a preliminary cross-sectional survey to investigate the prevalence of these left-behind children, their status of early childhood development and associated risk factors.

Methods A total of 1107 children were enrolled from two counties in Anhui province, China. Their caregivers completed questionnaires on demographics, the Early Childhood Development Questionnaire and the Parenting Self-efficacy Scale.

Results The estimated prevalence of left-behind children in the area was 86.6\% (959/1107), comprising 30.8\% (341/1107) and $55.8 \%(618 / 1107)$ children as a result of migration of either parent or both parents, respectively. The duration of breastfeeding for LBC was significant lower than those staying with mothers (8.74 vs. $10.14 ; \mathrm{p}<0.01)$. LBC had fewer reading books than non-left-behind children (NLBC) $(p<0.05)$. LBC also showed fewer times of physical check and higher case rate of diarrhoea in the last two weeks than NLBC. Male LBC who were fostered by caregivers with lower levels of parenting efficacy, and came from poorer families with less social support, experienced more mental health problems than other children.

Conclusions The study confirmed some negative outcomes for children who were left-behind in their early childhood. As the huge population proportion of LBC in rural China, more research on intervention strategies to improve the early development of LBC is urgently needed.

\section{0-208 RANDOMISED CONTROLLED TRIAL OF DAY CARE VERSUS HOSPITAL CARE OF SEVERE CHILDHOOD PNEUMONIA AND SEVERE MALNUTRITION IN DHAKA, BANGLADESGH}

${ }^{1} \mathrm{H}$ Ashraf, ${ }^{1} \mathrm{NH}$ Alam, ${ }^{2} \mathrm{~N}$ Gyr. ${ }^{1}$ Centre for Nutrition and Food Security, International Centre for Diarrhoeal Disease Research Bangladesh, Dhaka, Bangladesh; ${ }^{2}$ Faculty of Medicine, University of Basel, Basel, Switzerland

\subsection{6/archdischild-2014-307384.278}

Background and aims Management of severe pneumonia and malnutrition relies on hospital-based treatment but practical barriers often prevent children in areas with highest disease burden from receiving hospital care. A RCT compared clinical and cost of Day Care Approach (DCA) of management with that of hospital management.

Methods Children aged 2-59 months with severe pneumonia with severe malnutrition received either DCA at outpatient clinic with antibiotics, micronutrients, diet and supportive treatment from 08:00-17:00 daily, while mothers were educated on continuation of care at home, or hospital care with similar 24-hours treatment.
Results Four hundred seventy children aged 12.2 (8.8) months were equally assigned randomly to either DCA or hospital care, $13 \%$ had hepatomegaly and $11 \%$ had hypoxemia. The durations of day care and hospital were 7.9 (5.5) and 7.1 (3.1) days, respectively. Successful management was possible for $184 / 235$ [78.3\% (95\% CI 72.6- 83.1\%)] in DCA and 201/ 235 [85.5\% (95\% CI 80.5-89.5\%)] in hospital. Thirty-six [15.3\% (95\% CI 11.3-20.5\%)] in DCA and 22 [9.4\% (95\% CI 6.3-13.8\%)] in hospital required referral to hospitals. There were no deaths among DCA, however, 2 [0.9\% (95\% CI $0.2-3.0 \%)$ ] died in hospital. The average cost of treatment per episode per child was 165 US\$ for DCA and 256 US\$ for hospital.

Conclusions Severe pneumonia with severe malnutrition can be treated successfully and safely at reduced cost by DCA, but as effectively as hospital care, which can have an enormous impact in countries where recourses are scarce and hospital beds are limited for inpatient treatment.

\section{ESPNIC - Young Investigators Presentations}

\section{0-209 CONTINUOUS INCISIONAL LIDOCAINE INFUSION IN PAEDIATRIC PATIENTS FOLLOWING OPEN HEART SURGERY}

B Toledo, S Fernández, S Mencía, R González, E Sánchez, I López-Herce. Pediatric Intensive Care Unit, Hospital General Universitario Gregorio Marañón, Madrid, Spain

\subsection{6/archdischild-2014-307384.279}

Study objective To evaluate the efficacy of local incisional lidocaine infusion after heart surgery.

Methods Observational prospective study including post cardiac surgery children in whom a $0,5 \%$ lidocaine elastomer was intraoperatively placed within the surgical wound. Lidocaine doses and plasma levels $48 \mathrm{~h}$ after administration, adverse effects, additional analgesic or sedative drugs and analgesia and sedation score were registered. All patients received intravenous metamizol and paracetamol.

Results 105 patients were analysed. Median age was 66 months, with $21 \%$ of patients under 2 years of age. The most frequent surgical interventions were: Fontan surgery (26.2\%), atrial septal defects (20.4\%) and ventricular septal defects (10.7\%).

Median lidocaine administration duration was 58 h. $52.4 \%$ received fentanyl infusion (mean dose $1 \mathrm{mcg} / \mathrm{kg} / \mathrm{h}$, mean duration $24 \mathrm{~h}), 18.1 \%$ received midazolam infusion $(1.5 \mathrm{mcg} / \mathrm{kg} / \mathrm{h}, 8$ h) and $12 \%$ received propofol $(1.5 \mathrm{mg} / \mathrm{kg} / \mathrm{h}, 4 \mathrm{~h})$. Mean comfort score was 21.8 points and mean analgesia score was 1.9 (62\% of patients required additional analgesia).

Fentanyl infusion was less often needed in patients over 2 years old than in younger patients $(45.8 \%$ vs. $77.3 \%$; $\mathrm{p}=$ 0.015). Midazolam infusion was also less often required in patients over 2 years old $(13.3 \%$ vs $36.4 \%$; p $=0.025)$.

Lidocaine plasma levels were recorded $48 \mathrm{~h}$ after administration in 66 patients, $46 \%(\mathrm{n}=31)$ were within therapeutic range $(1.4-5 \mathrm{mcg} / \mathrm{ml})$. No important side - effects attributable to lidocaine were observed.

Conclusions Incisional analgesia with lidocaine is safe and effective after cardiac surgery. Children over 2 years of age require less additional analgesia and sedation. 